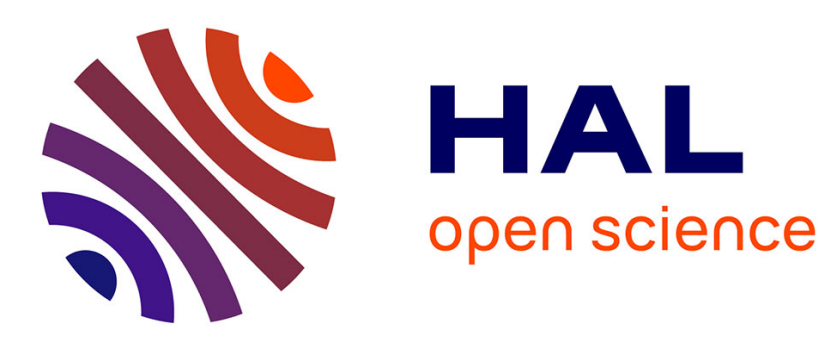

\title{
Kernel and Integral Representations of Operators on Infinite Dimensional Toy Fock Spaces \\ Yan Pautrat
}

\section{To cite this version:}

Yan Pautrat. Kernel and Integral Representations of Operators on Infinite Dimensional Toy Fock Spaces. Séminaire de Probabilités, 2006. hal-01735639

\section{HAL Id: hal-01735639 \\ https://hal.science/hal-01735639}

Submitted on 16 Mar 2018

HAL is a multi-disciplinary open access archive for the deposit and dissemination of scientific research documents, whether they are published or not. The documents may come from teaching and research institutions in France or abroad, or from public or private research centers.
L'archive ouverte pluridisciplinaire HAL, est destinée au dépôt et à la diffusion de documents scientifiques de niveau recherche, publiés ou non, émanant des établissements d'enseignement et de recherche français ou étrangers, des laboratoires publics ou privés. 


\title{
Kernel and Integral Representations of Operators on Infinite Dimensional Toy Fock Spaces
}

\author{
Yan Pautrat
}

Department of Mathematics and Statistics, McGill University, 805 Sherbrooke Street West, Montreal, QC, H3A 2K6, Canada.

e-mail: pautrat@math.mcgill.ca

Summary. We study conditions for the existence of a Maassen kernel representation for operators on infinite dimensional toy Fock spaces. When applied to the toy Fock space of discrete quantum stochastic calculus, this condition gives a criterion for the existence of a representation as a quantum stochastic integral, as well as explicit formulas for deriving the coefficients of this representation.

Key words: Fock space, toy Fock space, Maassen-Meyer representation, quantum stochastic integration

\section{Introduction}

The representation of operators on Fock space over $L^{2}\left(\mathbb{R}_{+}\right)$or $L^{2}\left(\mathbb{R}_{+}^{d}\right)$ as Maassen-Meyer kernel operators - that is, as series of iterated integrals of scalars with respect to quantum noises - is of particular interest. Indeed, this type of representation has been heavily studied because kernel operators naturally arise as solutions of quantum stochastic differential equations (see [Maa], [Mey]); what is more, composing kernel operators gives a kernel operator which can be explicitly computed. Nevertheless, there is no satisfactory criterion for representability of an operator, as existing results are scarce (see [At1], [B-L]).

The purpose of this article is to give very general representability criteria in the simpler case of infinite dimensional toy Fock space, that is, antisymmetric Fock space over $\ell^{2}(\mathbb{N})$ or $\ell^{2}\left(\mathbb{N}^{d}\right)$. The search for such results was motivated by Attal's rigorous method to approximate boson Fock space over $L^{2}\left(\mathbb{R}_{+}\right)$(which we denote by $\Phi)$ by its "discrete-time" counterpart: infinite dimensional toy Fock space T $\Phi$ (see [At2]). Since the approximation method is explicit, finding criteria for the representability of operators on T $\Phi$ could lead to representations as kernel operators (and, as we shall see later, as quantum stochastic integrals) of approximations of operators on $\Phi$. 
Furthermore, the toy Fock space T $\Phi$ conspicuously has interesting features regarding representability as kernel operators: indeed it is straightforward to see that the von Neumann subalgebra of $\mathcal{B}(\mathrm{T} \Phi)$ (the set of bounded operators on T $\Phi$ ) generated by all fundamental operators $a_{i}^{+}, a_{i}^{-} a_{i}^{\circ}, i \geqslant 0$, is $\mathcal{B}(\mathrm{T} \Phi)$ itself. This leads to some kind of kernel representability result for bounded operators of T $\Phi$. Another simple approach, presented in section 2, gives a more precise result on the representation of a bounded operator; yet this result is still unsatisfactory both in its range of application (the operators considered in physical practice are rarely bounded) and in the underlying meaning given to a kernel representation. In this paper we discuss a more satisfactory definition of such a representation. We then search equivalent formulations of that definition; we will see that, with minor domain assumptions, these formulations lead to explicit formulas for the coefficients involved in the kernel and yield a very general sufficient condition for the existence of a kernel representation. The proofs use discrete-time analogues of continuous-time methods developed by Lindsay in [Lin]; we point out along the way a mistake contained in one of the propositions of that paper, give a counterexample and make a tentative correction. Later on we apply our results on kernel representations to obtain criteria and formulas for representations of operators as quantum stochastic integrals.

This paper is organized as follows: in section 1 we give all the needed notations. In section 2 we fulfill the above program on kernel representations. In section 3 we apply our kernel representation theorems to the study of quantum stochastic integral representations.

\section{Notations}

In the purpose of generalizing our representability theorems to criteria that apply in any antisymmetric Fock space over an infinite-dimensional, separable Hilbert space, we introduce all notations in a general framework. Denote by $\mathcal{A}$ an arbitrary infinite countable set, and by $\mathcal{P}$ the set of all finite subsets of $\mathcal{A}$. The space we will work on, throughout this paper, is the antisymmetric Fock space over $\ell^{2}(\mathcal{A})$, which, by Guichardet's interpretation, can be seen as $\ell^{2}(\mathcal{P})$, that is, the space of all maps $f: \mathcal{P} \mapsto \mathbb{C}$, such that

$$
\sum_{M \in \mathcal{P}}|f(M)|^{2}<+\infty .
$$

The most familiar case is $\mathcal{A}=\mathbb{N}$, where $\ell^{2}(\mathcal{P})$ is the infinite dimensional toy Fock space.

Let us denote by $X_{A}$ the indicator function of $A \in \mathcal{P}$; the set of all $X_{A}$ 's constitutes a (Hilbertian) basis of $\ell^{2}(\mathcal{P})$. This particular basis being fixed, one defines for all $B \in \mathcal{P}$ three operators by 


$$
\begin{aligned}
& a_{B}^{+} X_{A}=\left\{\begin{array}{cl}
X_{A \cup B} & \text { if } B \cap A=\varnothing \\
0 & \text { otherwise, }
\end{array}\right. \\
& a_{B}^{-} X_{A}=\left\{\begin{array}{cl}
X_{A \backslash B} & \text { if } B \subset A \\
0 & \text { otherwise, }
\end{array}\right. \\
& a_{B}^{\circ} X_{A}=\left\{\begin{array}{cl}
X_{A} & \text { if } B \subset A \\
0 & \text { otherwise. }
\end{array}\right.
\end{aligned}
$$

Those operators are called respectively the creation, annihilation and conservation operators. From now on, for two subsets $A, B$ of $\mathcal{A}$, we write

- $A+B$ for $A \cup B$ if $A \cap B=\varnothing$,

- $A-B$ for $A \backslash B$ if $B \subset A$,

using the convention that any quantity in which $A+B$ (respectively $A-B$ ) appears as a variable or as an index is null if $A \cap B \neq \varnothing$ (respectively $B \not \subset A$ ). We will write for example

$$
\begin{aligned}
& a_{B}^{+} X_{A}=X_{A+B}, \\
& a_{B}^{-} X_{A}=X_{A-B} .
\end{aligned}
$$

Practically this will turn out to be a short notation to restrict the range of summation of sums in which the variables are elements of $\mathcal{P}$.

Let us write the general expression of the action of an operator $a_{A}^{+} a_{B}^{\circ} a_{C}^{-}$on a vector $f$ of $\ell^{2}(\mathcal{A})$. First, if $A, B, C$ are not mutually disjoint then $a_{A}^{+} a_{B}^{\circ} a_{C}^{-}$ is null; otherwise for all $M$ in $\mathcal{P}$,

$$
a_{A}^{+} a_{B}^{\circ} a_{C}^{-} f(M)=f(M+C-A)
$$

if $B \subset M$, and zero otherwise.

Remark that we have not included here a precise definition of what we will call a kernel representation. We postpone this and the preparative discussion to the next section.

\section{Kernel representation theorems on $\ell^{2}(\mathcal{P})$}

Let us describe a tentative approach to representations of bounded operators; to that end let us restrict our framework to the (ordered) case where $\mathcal{A}=\mathbb{N}$, and denote by $p_{i}$ the orthogonal projection on the subset $\ell^{2}\left(\mathcal{P}_{i}\right)$, where $\mathcal{P}_{i}$ is the set of subsets of $\{0, \ldots, i-1\}$. In that case, for any bounded operator $K$ on $\ell^{2}(\mathcal{P}), p_{i} K p_{i}$ is an operator on $\ell^{2}\left(\mathcal{P}_{i}\right)$, and the sequence $\left(p_{i} K p_{i}\right)_{i \geqslant 0}$ converges strongly to $K$. It is known from the case of finite dimensional toy Fock spaces that every one of these $p_{i} K p_{i}$ coincides with a kernel operator $p_{i} \sum_{A, B, C<i} k_{i}(A, B, C) a_{A}^{+} a_{B}^{\circ} a_{C}^{-} p_{i}$. It is easy to see that the $k_{i}$ 's are compatible in the sense that there exists a kernel $k: \mathcal{P}^{3} \mapsto \mathbb{C}$ which extends all kernels $k_{i}$. 
This $k$ is such that for any vector $f \in \ell^{2}(\mathcal{P})$, any $M \in \mathcal{P}$,

$$
(K f)(M)=\lim _{i \rightarrow \infty} \sum_{U+V+W=M} \sum_{N \in \mathcal{P}_{i}} k(U, V, N) f(V+W+N),
$$

so that, in some sense, the function $k$ satisfies an analogue of the formula which defines kernel operators in continuous time (see [Mey], [Maa]). Nevertheless, let us discuss what we want a kernel representation to be. Heuristically, such a representation should be a series $\sum_{(A, B, C) \in \mathcal{P}^{3}} k(A, B, C) a_{A}^{+} a_{B}^{\circ} a_{C}^{-}$, the meaning of the sum being taken in a weak sense: we expect that, for every $f$ in some domain, the formal computation $\sum_{A, B, C} k(A, B, C) a_{A}^{+} a_{B}^{\circ} a_{C}^{-} \sum_{N} f(N) X_{N}$, gives the right result at every $M$ in $\mathcal{P}$, that is, that the equality

$$
(K f)(M)=\sum_{U+V+W=M} \sum_{N \in \mathcal{P}} k(U, V, N) f(V+W+N),
$$

holds for every $N$ in $\mathcal{P}$. It is a sensible demand, both from a pragmatic point of view and from an intuitive one (the sums $\sum_{A, B, C}$ and $\sum_{N}$ above should be independent of the order of summation, so the final one also should) that this series be absolutely convergent. In equation (2) the series a priori lacks that property. It is therefore natural to look for conditions upon which that property holds.

Returning to the general case (where $\mathcal{A}$ is any infinite countable set), we will solve this problem and obtain further representation theorems. The strategy of our proof will be completely different from the above; our basic tools will be the following:

- the transform $k \mapsto k^{\prime}$ similar to the one defined by Lindsay (see [Lin]) in the regular Fock space; we give its definition below.

- The additional feature, specific to the case of discrete-time, of equivalence of two variables and three variables representation. This feature is the simple fact that, since any $a_{B}^{\circ}$ is $a_{B}^{+} a_{B}^{-}$, one should (and does, as Proposition 1 will prove) obtain equivalent actions for the formal series $\sum_{(A, B, C) \in \mathcal{P}^{3}} k(A, B, C) a_{A}^{+} a_{B}^{\circ} a_{C}^{-}$and $\sum_{(A, B) \in \mathcal{P}^{2}} k(A, B) a_{A}^{+} a_{B}^{-}$if we use the correspondence

$$
k(A, B)=k(A \backslash B, A \cap B, B \backslash A) \quad \text { and } \quad k(A, B, C)=k(A \cup B, B \cup C) .
$$

Definition 1. For a function $k: \mathcal{P}^{3} \mapsto \mathbb{C}$, let us define $k^{\prime}: \mathcal{P}^{3} \mapsto \mathbb{C}$ as

$$
k^{\prime}(A, B, C)=\sum_{V \subset B} k(A, V, C),
$$

and for a function $k: \mathcal{P}^{2} \mapsto \mathbb{C}$, let us define $k^{\prime}: \mathcal{P}^{2} \mapsto \mathbb{C}$ as

$$
k^{\prime}(A, B)=\sum_{V \subset A \cap B} k((A \backslash B) \cup V,(B \backslash A) \cup V) .
$$


Remark. For subsets $A, B, C$ which are not mutually disjoint, the operator $a_{A}^{+} a_{B}^{\circ} a_{C}^{-}$is null; therefore, when considering kernels with three arguments, the function $k$ needs only to be defined on mutually disjoint triples for our purpose, and similarly for $k^{\prime}$.

Properties of the transform

- The correspondence $k \mapsto k^{\prime}$ is bijective for both three-arguments and twoarguments kernels thanks to the Moebius inversion formula which yields

$$
k(A, B, C)=\sum_{V \subset B}(-1)^{|B-V|} k^{\prime}(A, V, C)
$$

and

$$
k(A, B)=\sum_{V \subset A \cap B}(-1)^{|A \cap B-V|} k^{\prime}((A \backslash B) \cup V,(B \backslash A) \cup V) .
$$

- The correspondence defined by (4) is bijective between the set of functions defined on the subset of $\mathcal{P}^{3}$ of mutually disjoint triples of $\mathcal{P}$ and the set of functions on $\mathcal{P}^{2}$

- If, in the next few lines, we add indices and denote kernels by $k_{2}, k_{2}^{\prime}$, $k_{3}, k_{3}^{\prime}$, depending on the number of variables, the following diagram is commutative:<smiles>[Y]1ncn[nH]1</smiles>

where arrows are either the correspondence in (4) or the transformation in Definition 1. That means in particular that notations like $k_{2}^{\prime}, k_{3}^{\prime}$ would be unambiguous.

- Our choice for the transform $k \mapsto k^{\prime}$ extends the equalities in (4) to the functions $k^{\prime}$ :

for mutually disjoint $(A, B, C) \in \mathcal{P}^{3}, \quad k^{\prime}(A, B, C)=k^{\prime}(A \cup B, B \cup C)$.

Because of these properties we will not distinguish anymore notations between $k_{2}$ and $k_{3}$, nor between $k_{2}^{\prime}$ and $k_{3}^{\prime}$.

The following proposition contains the first properties that link the four different forms of a kernel.

Proposition 1. Let $f$ be a fixed vector in $\ell^{2}(\mathcal{A})$. Define the four assumptions:

- for all mutually disjoint $U, V, W$ in $\mathcal{P}$

$$
\sum_{N \in \mathcal{P}}|k(U, V, N) f(V+W+N)|<+\infty
$$


- for all disjoint $U, V$ in $\mathcal{P}$

$$
\sum_{N \in \mathcal{P}}|k(U, N) f(V+N)|<+\infty
$$

- for all disjoint $U, V$ in $\mathcal{P}$

$$
\sum_{N \in \mathcal{P}}\left|k^{\prime}(U, V, N) f(V+N)\right|<+\infty
$$

- for all $U$ in $\mathcal{P}$

$$
\sum_{N \in \mathcal{P}}\left|k^{\prime}(U, N) f(N)\right|<+\infty .
$$

Then the conditions on two-arguments kernels are equivalent to their threearguments counterparts, that is, (7) and (8) are equivalent, (9) and (10) are equivalent. What is more, the conditions on the kernels imply the conditions on their transforms, that is, (7), (8) imply (9), (10).

Besides, if all conditions are satisfied, then the following are defined and equal for all $M \in \mathcal{P}$ :

$$
\begin{array}{cl}
\sum_{U+V+W=M} & \sum_{N} k(U, V, N) f(V+W+N) \\
\sum_{U+V=M} & \sum_{N} k^{\prime}(U, V, N) f(V+N) \\
\sum_{U+V=M} & \sum_{N} k(U, N) f(V+N) \\
& \sum_{N} k^{\prime}(M, N) f(N)
\end{array}
$$

Proof. Let us start with the proof that (7) implies (8): first fix $U_{0}, V_{0}$;

$$
\begin{aligned}
\sum_{N} \mid k\left(U_{0},\right. & N) f\left(V_{0}+N\right) \mid \\
& =\sum_{N}\left|k\left(U_{0} \backslash N, U_{0} \cap N, N \backslash U_{0}\right) f\left(V_{0}+U_{0} \cap N+N \backslash U_{0}\right)\right| \\
& \leqslant \sum_{U+V=U_{0}} \sum_{N}\left|k\left(U, V, N \backslash U_{0}\right) f\left(V+V_{0}+N \backslash U_{0}\right)\right| \\
& \leqslant 2^{\left|U_{0}\right|} \sum_{U+V=U_{0}} \sum_{N \text { disjoint from } U, V}\left|k(U, V, N) f\left(V+V_{0}+N\right)\right| \\
& \leqslant 2^{\left|U_{0}\right|} \sum_{U+V=U_{0}} \sum_{N}\left|k(U, V, N) f\left(V+V_{0}+N\right)\right| \\
& <+\infty
\end{aligned}
$$


where the $2^{\left|U_{0}\right|}$ arises because any $N$ can be written as at most $2^{\left|U_{0}\right|}$ different " $N \backslash U_{0}$ ".

Now prove that (8) implies (7):

$$
\begin{aligned}
\sum_{N}|k(U, V, N) f(V+W+N)| & =\sum_{N}|k(U+V, N+V) f(W+(N+V))| \\
& =\sum_{N \supset V}|k(U+V, N) f(W+N)| \\
& <+\infty .
\end{aligned}
$$

The equivalence of (9) and (10) is shown exactly in the same way.

To show that the above conditions on $k$ imply those on the transform $k^{\prime}$, we prove that (7) implies (9):

$$
\sum_{N}\left|k^{\prime}(U, V, N) f(V+N)\right| \leqslant \sum_{N} \sum_{\alpha \subset V}|k(U, \alpha, N) f(\alpha+(V \backslash \alpha)+N)|
$$

and the right-hand side is just a finite sum of series of the type (7) with $(U, V, W)=(U, \alpha, V \backslash \alpha)$.

The equalities are obvious once the summability assumptions allow all manipulations on the sums.

Remark. It is not true in general that the conditions on $k^{\prime}$ imply their counterpart on $k$. Here is a counterexample: let $k$ be of the form

$$
k(U, V, W)=(-1)^{|V|} j(U, W)
$$

for some function $j$ of two disjoint finite subsets of $\mathbb{N}$, which is not to be confused with the kernel $k$ expressed as function of two variables.

Then $k^{\prime}(U, V, W)=\mathbb{1}_{V=\varnothing} j(U, W)$ and (9) simply becomes

$$
\text { for all } U \in \mathcal{P}, \quad \sum_{N}|j(U, N) f(N)|<+\infty,
$$

whereas (7) is

$$
\text { for all }(U, V) \in \mathcal{P}^{2}, \quad \sum_{N}|j(U, N) f(V+N)|<+\infty .
$$

Now if one considers

- a function $j$ such that $j(U, W)=0$ if the cardinality of $W$ is different from 1 ,

- a vector $f$ null on sets of cardinality one,

then (15) is trivial while (16) becomes 


$$
\text { for all }(U, V) \in \mathcal{P}^{2}, \quad \sum_{n \geqslant 0}|j(U,\{n\}) f(V+\{n\})|<+\infty \text {. }
$$

and still implies a condition on the values of $j$ and $f$, so that many counterexamples exist.

The same type of counterexamples holds in continuous time for the equivalence described by Lindsay ([Lin]). The next theorem describes a class of vectors for which the equivalence of $(7),(8),(9),(10)$ holds; once again this class translates in continuous time to a class of vectors for which the equivalence described by Lindsay holds.

Theorem 1. Let $f$ be a vector in $\ell^{2}(\mathcal{P})$ for which there exists a function $\phi: \mathcal{A} \mapsto \mathbb{R}_{+}$such that

$$
\text { for all }(A, B) \in \mathcal{P}^{2}, \quad|f(A+B)| \leqslant|f(A)| \prod_{i \in B} \phi(i) .
$$

Then assumptions (7), (8), (9), (10) are equivalent for that $f$.

Proof. What is left to prove is that (9) implies (7). Using the particular hypothesis on $f$, one has for all $U, V, W$,

$$
\begin{aligned}
\sum_{N}|k(U, V, N) f(V+W+N)| & \leqslant \prod_{i \in W} \phi(i) \sum_{N_{\text {disjoint from } V, W}|k(U, V, N) f(V+N)|} \\
& \leqslant \prod_{i \in W} \phi(i) \sum_{N}|k(U, V, N) f(V+N)|
\end{aligned}
$$

so we can reduce the proof to the case where $W=\varnothing$. But in that case, using the inverse Moebius transform,

$$
\begin{aligned}
\sum_{N}|k(U, V, N) f(V+N)| & \leqslant \sum_{\alpha \subset V} \sum_{N}\left|k^{\prime}(U, \alpha, N) f(V+N)\right| \\
& \leqslant \sum_{\alpha \subset V} \sum_{N}\left|k^{\prime}(U, \alpha, N) f(\alpha+N)\right| \prod_{i \in V \backslash \alpha} \phi(i) \\
& <+\infty
\end{aligned}
$$

Definition 2. The vectors which satisfy the property mentioned in the previous theorem are called subexponential. The set of all subexponential vectors, which we denote by $s \mathcal{E}$, contains all linear combinations of exponential vectors or vectors $X_{A}, A \in \mathcal{P}$.

The set $s \mathcal{E}$ is not a vector space; nevertheless, it has the properties that, if $f, g$ are in $s \mathcal{E}$ and $\lambda, \mu$ are in $\mathbb{C}$, then $|\lambda||f|+|\mu||g|$ is in $s \mathcal{E}$.

Here is a precise definition, following our earlier discussion, of what we call a kernel operator: 
Definition 3. $A$ (possibly unbounded) operator $K$ on $\ell^{2}(\mathcal{P})$ is said to have a kernel representation if there exists a function $k$ such that:

- Dom $K$ is exactly the set of $f \in \ell^{2}(\mathcal{P})$ that satisfy one of the conditions (7) or $(8)$,

- the equalities in (11), (12), (13) or (14) define a square integrable function of $M$,

- $K f(M)$ is equal to the corresponding expression for all $M \in \mathcal{P}$.

Now, up to an additional simple assumption, the kernel decomposition takes a clear meaning: suppose that an operator $K$ has such a representation and that the basis $\left\{X_{A}\right\}$ is in $\operatorname{Dom} K$, then writing $\sum_{N} k^{\prime}(M, N) \mathbb{1}_{A}(N)=$ $\left(K X_{A}\right)(M)$ yields the fundamental formula

$$
\left\langle X_{M}, K X_{A}\right\rangle=k^{\prime}(M, A) .
$$

Thanks to this formula, (10) becomes

$$
\forall M, \quad \sum_{N}\left|\left\langle X_{M}, K X_{N}\right\rangle f(N)\right|<+\infty,
$$

and the other two assumptions (square-integrability and equality of expressions) simply mean that one can write rigourously

$$
\forall M, \quad\left\langle X_{M}, K \sum_{N} f(N) X_{N}\right\rangle=\sum_{N}\left\langle X_{M}, K X_{N}\right\rangle f(N) .
$$

What this means indeed is that kernel representations are just another way to write the above expansion. Of course there are conditions for this expansion to be meaningful and conditions for the obtained representation to actually represent the original operator. We discuss these conditions after the following proposition:

Proposition 2. Let $K$ be an operator with domain such that

$$
\left\{X_{A}, A \in \mathcal{P}\right\} \subset \operatorname{Dom} K \subset s \mathcal{E} .
$$

Then $K$ can be extended to a kernel operator if and only if for every $f \in$ Dom $K$ and every $M$ in $\mathcal{P}$,

$$
\left\{\begin{array}{c}
\sum_{N}\left|\left\langle X_{M}, K X_{N}\right\rangle f(N)\right|<+\infty \text { and } \\
\sum_{N}\left\langle X_{M}, K X_{N}\right\rangle f(N)=K f(M) .
\end{array}\right.
$$

In that case, the associated kernel is given by

$$
k^{\prime}(A, B)=\left\langle X_{A}, K X_{B}\right\rangle .
$$

Proof. Thanks to Proposition 1, Theorem 1 and formula (18), this is a simple rephrasing of our definition. 
It is clear that, if the domain of the operator $K$ is not contained in the subexponential subset, then the obtained kernel operator can be such that $\left\{X_{A}\right\} \subsetneq \operatorname{Dom} K_{k}$ and $\left\{X_{A}\right\}=\operatorname{Dom} K \cap \operatorname{Dom} K_{k}$.

It is also clear that, even if the conditions

$$
\begin{gathered}
\sum_{N}\left|\left\langle X_{M}, K X_{N}\right\rangle f(N)\right|<+\infty \\
\sum_{M}\left|\sum_{N}\left\langle X_{M}, K X_{N}\right\rangle f(N)\right|^{2}<+\infty
\end{gathered}
$$

hold for any $f$ in Dom $K$, so that the associated kernel operator $K_{k}$ is well defined on Dom $K$ and coincides with $K$ on $\left\{X_{A}, A \in \mathcal{P}\right\}$, one needs a kind of closability assumption to make sure that the kernel operator is indeed an extension of $K$ : this assumption is exactly the condition

$$
\sum_{N}\left\langle X_{M}, K X_{N}\right\rangle f(N)=K f(M)
$$

It does not seem that a more concise formulation can be found: the usual closability property would be that if a sequence $\left(u_{n}\right)_{n \geqslant 0}$ converges to zero and is such that the sequence of images $\left(K u_{n}\right)_{n \geqslant 0}$ is convergent, then its limit is zero.

Assumption (19) is weaker than closability in the sense that it only considers approximating sequences $\left(u_{n}\right)_{n \geqslant 0}$ made of partial sums of $\sum_{N} f(N) X_{N}$, but also stronger than closability in the sense that convergence to zero of $\left(u_{n}\right)_{n \geqslant 0}$ with the weak convergence assumption on the images that $\left\langle X_{M}, K u_{n}\right\rangle$ converges for all $M$ and defines a square-integrable function of $M$ must imply that that limit is zero.

On the other hand, it is clear that these properties are satisfied if one assumes that $K$ has an adjoint defined on all vectors $X_{M}, M$ in $\mathcal{P}$. Indeed in that case, we have the following result. Note that no assumption of the type Dom $K \subset s \mathcal{E}$ is needed; actually the proof of this theorem proves the stronger summability assumptions on the kernels $k$ themselves and not on the transforms $k^{\prime}$.

Theorem 2. Let $K$ be an operator on $\ell^{2}(\mathcal{P})$ such that the set of all $X_{A}$ is in Dom $K \cap \operatorname{Dom} K^{*}$. Then the kernel operator defined by (18) is a closed extension of $K$.

Proof. Let us define the kernel $k$ by (18). We will show that assumption (7) holds for any vector $f$ of $\ell^{2}(\mathcal{P})$. Indeed, let us fix $U, V, W$; then from the Moebius inversion formula, one has for all $N$ disjoint from $U, V, W$ : 


$$
\begin{aligned}
|k(U, V, N)| & \leqslant \sum_{\alpha \subset V}\left|k^{\prime}(U+\alpha, N+\alpha)\right| \\
& \leqslant \sum_{\alpha \subset V}\left|\left\langle X_{U+\alpha}, K X_{N+\alpha}\right\rangle\right| \\
& \leqslant \sum_{\alpha \subset V}\left|\left\langle K^{*} X_{U+\alpha}, X_{N+\alpha}\right\rangle\right|,
\end{aligned}
$$

which is a finite sum of square-summable terms. Besides, $N \mapsto f(V+W+N)$ is also square-summable, and therefore $\sum_{N}|k(U, V, N) f(V+W+N)|$ is finite.

The condition on $f$ appears in the sequel: the domain of the kernel operator $K_{k} f$ is the set of vectors $f$ in T $\Phi$ such that

$$
K_{k} f(M)=\sum_{N}\left\langle X_{M}, K X_{N}\right\rangle f(N)
$$

defines a function belonging to $\ell^{2}(\mathcal{P})$. That quantity is equal to

$$
\sum_{N}\left\langle K^{*} X_{M}, X_{N}\right\rangle f(N)=\left\langle K^{*} X_{M}, f\right\rangle
$$

so that the domain of $K_{k}$ is the set of vectors $f$ such that $M \mapsto\left\langle K^{*} X_{M}, f\right\rangle$ is square-integrable. For all $f$ in $\operatorname{Dom} \bar{K},\left\langle K^{*} X_{M}, f\right\rangle=\left\langle X_{M}, \bar{K} f\right\rangle$, so $\operatorname{Dom} \bar{K} \subset$ Dom $K_{k}$ and $K_{k} f=\bar{K} f$.

We now prove that $K_{k}$ is closed: let $\left(f_{n}\right)_{n \geqslant 0}$ be a sequence in Dom $K_{k}$ that converges to some $f$ in T $\Phi$ and such that $\left(K_{k} f_{n}\right)_{n \geqslant 0}$ converges to some $\phi$ in TФ. Then

$$
\begin{aligned}
\sum_{M}\left|\left\langle K^{*} X_{M}, f\right\rangle\right|^{2} & =\sum_{M} \lim \left|\left\langle K^{*} X_{M}, f_{n}\right\rangle\right|^{2} \\
\sum_{M} \lim \left|\left\langle K^{*} X_{M}, f_{n}\right\rangle\right|^{2} & \leqslant \liminf \sum_{M}\left|\left\langle K^{*} X_{M}, f_{n}\right\rangle\right|^{2} \\
\liminf \sum_{M}\left|\left\langle K^{*} X_{M}, f_{n}\right\rangle\right|^{2} & =\liminf \left\|K_{k} f_{n}\right\|^{2} \\
\liminf \left\|K_{k} f_{n}\right\|^{2} & =\|\phi\|^{2} \\
\|\phi\|^{2} & <+\infty,
\end{aligned}
$$

so that $f$ lies in Dom $K_{k}$; besides

$$
\begin{aligned}
K_{k} f(M) & =\left\langle K^{*} X_{M}, f\right\rangle \\
\left\langle K^{*} X_{M}, f\right\rangle & =\lim \left\langle K^{*} X_{M}, f_{n}\right\rangle \\
\lim \left\langle K^{*} X_{M}, f_{n}\right\rangle & =\lim \left\langle X_{M}, K_{k} f_{n}\right\rangle \\
\lim \left\langle X_{M}, K_{k} f_{n}\right\rangle & =\phi(M)
\end{aligned}
$$

holds for every $M$ in $\mathcal{P}$ so that $\phi=K_{k} f$ and the proof is complete.

Remark. The inclusion $\bar{K} \subset K_{k}$ is a priori not an equality.

This theorem gives numerous examples of operators that admit a kernel representation. 


\section{Integral representation of operators on toy Fock space}

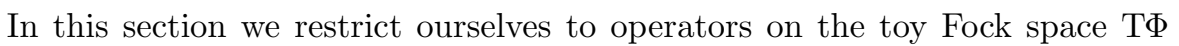
of quantum stochastic calculus, for which $\mathcal{A}=\mathbb{N}$; indeed we wish to consider integrals of operators, and this demands a natural ordering on our set $\mathcal{A}$. Let us suppose that an operator $K$ on $\mathrm{T} \Phi$ has a kernel representation in the sense of Definition 3. This kernel representation is formally an expression of $K$ as $\sum_{A, B, C} k(A, B, C) a_{A}^{+} a_{B}^{\circ} a_{C}^{-}$. Let us go on with formal expressions and apply careless manipulations to this sum: we write for all $(A, B, C) \neq(\varnothing, \varnothing, \varnothing)$ $a_{A}^{+} a_{B}^{\circ} a_{C}^{-}=a_{A \backslash i}^{+} a_{B}^{\circ} a_{C}^{-} a_{i}^{+}$when e.g. the largest element $i$ in $A \cup B \cup C$ is in $A$, and regroup terms. We obtain

$$
\begin{aligned}
& k(\varnothing, \varnothing, \varnothing)+\sum_{i} \sum_{A, B, C<i} k(A+i, B, C) a_{A}^{+} a_{B}^{\circ} a_{C}^{-} a_{i}^{+} \\
& \quad+\sum_{i} \sum_{A, B, C<i} k(A, B+i, C) a_{A}^{+} a_{B}^{\circ} a_{C}^{-} a_{i}^{\circ}+\sum_{i} \sum_{A, B, C<i} k(A, B, C+i) a_{A}^{+} a_{B}^{\circ} a_{C}^{-} a_{i}^{-},
\end{aligned}
$$

that is, we obtain an integral representation of $K$ with the following integrands:

$$
\left\{\begin{aligned}
k_{i}^{+} & =\sum_{A, B, C<i} k(A+i, B, C) a_{A}^{+} a_{B}^{\circ} a_{C}^{-} \\
k_{i}^{\circ} & =\sum_{A, B, C<i} k(A, B+i, C) a_{A}^{+} a_{B}^{\circ} a_{C}^{-} \\
k_{i}^{-} & =\sum_{A, B, C<i} k(A, B, C+i) a_{A}^{+} a_{B}^{\circ} a_{C}^{-}
\end{aligned}\right.
$$

Giving a more rigorous meaning to that demands, of course, a definition of the integrals. The definitions are actually quite simple (see $[\mathrm{Pau}])$ : the domain of a sum $\sum_{i} h_{i}$ is the set of vectors $f$ such that for all $A \in \mathcal{P}$, the series $\sum_{i} h_{i} f(A)$ is summable and defines a square-integrable function of $A$. An integral $\sum_{i} h_{i}^{\epsilon} a_{i}^{\epsilon}$ is such a sum with $h_{i}=h_{i}^{\epsilon} a_{i}^{\epsilon}$; on the other hand, one can see from Definition 3 and from the expression (1) that a kernel representation is simply a series $\sum_{A, B, C}\left(k(A, B, C) a_{A}^{+} a_{B}^{\circ} a_{C}^{-}\right)$in the above sense. This justifies the above manipulations except for domain properties; what it exactly yields is

$$
K \subset k(\varnothing, \varnothing, \varnothing)+\sum_{i}\left(k_{i}^{+} a_{i}^{+}+k_{i}^{\circ} a_{i}^{\circ}+k_{i}^{-} a_{i}^{-}\right),
$$

which does not prove in our sense that our operator has an integral representation, since the right-hand side is itself an extension of the integral

$$
k(\varnothing, \varnothing, \varnothing)+\sum_{i} k_{i}^{+} a_{i}^{+}+\sum_{i} k_{i}^{\circ} a_{i}^{\circ}+\sum_{i} k_{i}^{-} a_{i}^{-} .
$$

What we have shown is that on some domain (the intersection of the domain of this integral and the domain of the operator), the operator coincides with the integral. Moreover, if a vector $X_{A}$ is in the domain of $K$, then it is also in the domain of the integral (21), since only one of the three series $\sum_{i} k_{i}^{\epsilon} a_{i}^{\epsilon} X_{A}$ has an infinite number of nonzero terms. 
The interesting aspect of this formal computation is that the integrands defined by (20) are everywhere defined as finite sums of everywhere defined operators, and are described as simple kernel operators with explicit kernels. Using the formula (18) then gives the action of the operators $k_{i}^{\epsilon}$ expressed as functions of $K$ :

$$
\left\{\begin{array}{l}
\left\langle X_{A}, k_{i}^{+} X_{B}\right\rangle=\mathbb{1}_{A \Delta B<i}\left\langle X_{A_{i[}+i}, K X_{B_{i[}}\right\rangle \\
\left\langle X_{A}, k_{i}^{-} X_{B}\right\rangle=\mathbb{1}_{A \Delta B<i}\left\langle X_{A_{i[}}, K X_{B_{i[}+i}\right\rangle \\
\left\langle X_{A}, k_{i}^{\circ} X_{B}\right\rangle=\mathbb{1}_{A \Delta B<i}\left(\left\langle X_{A_{i[}+i}, K X_{B_{i[}+i}\right\rangle-\left\langle X_{A_{i[}}, K X_{B_{i[}}\right\rangle\right)
\end{array}\right.
$$

Using the fundamental operators of abstract Ito calculus on toy Fock space $p_{i}, d_{i}$, these operators have much more interesting expressions. We recall briefly from [Pau] the definitions of these operators: for $f \in \mathrm{T} \Phi$ and $i \in \mathbb{N}$, $p_{i} f$ and $d_{i} f$ are defined as the vectors of T $\Phi$ such that, for $A \in \mathcal{P}$,

$$
\begin{aligned}
& p_{i} f(M)=\mathbb{1}_{M<i} f(M), \\
& d_{i} f(M)=\mathbb{1}_{M<i} f(M+i) .
\end{aligned}
$$

In particular,

$$
\begin{aligned}
p_{i} X_{A} & =\mathbb{1}_{A<i} X_{A}, \\
d_{i} X_{A} & =\mathbb{1}_{A<i} X_{A-i},
\end{aligned}
$$

from which one sees immediately that one has

$$
\left\{\begin{aligned}
k_{i}^{+} & p_{i}=d_{i} K p_{i} \\
k_{i}^{-} & p_{i}=p_{i} K a_{i}^{+} p_{i} \\
k_{i}^{\circ} & p_{i}=d_{i} K a_{i}^{+}-p_{i} K p_{i}
\end{aligned}\right.
$$

The above discussion proves the following result:

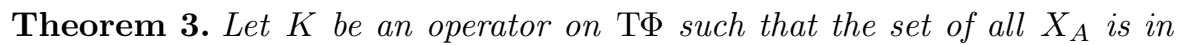
$\operatorname{Dom} K \cap \operatorname{Dom} K^{*}$. Then the operator

$$
\lambda+\sum_{i} k_{i}^{+} a_{i}^{+}+\sum_{i} k_{i}^{\circ} a_{i}^{\circ}+\sum_{i} k_{i}^{-} a_{i}^{-}-K,
$$

where the operators $k_{i}^{\epsilon}$ are defined by $(22)$ and $\lambda=\langle\mathbb{1}, K \mathbb{1}\rangle$, is a restriction of the zero process and its domain contains the set $\left\{X_{A}, A \in \mathcal{P}\right\}$.

The $p_{i}$ 's on the right of expressions in (22) are here only for the sake of symmetry; if one notices that $d_{i}=p_{i} a_{i}^{-}$and that $a_{i}^{+}, a_{i}^{-}$are mutually adjoint, these formulas show that $\left(k^{*}\right)_{i}^{+}=k_{i}^{-},\left(k^{*}\right)_{i}^{-}=k_{i}^{+}$and $\left(k^{*}\right)_{i}^{\circ}=k_{i}^{\circ}$, where the $\left(k^{*}\right)_{i}^{\epsilon}$ are the integrands in the integral representation of $K^{*}$. 


\section{References}

[At1] S. Attal: Non-commutative chaotic expansion of Hilbert-Schmidt operators on Fock space, Comm. in Math. Phys. Vol. 175, Springer, Berlin, 1996, pp. 43-62.

[At2] S. AtTal: Approximating the Fock space with the toy Fock space, Sém. de Probabilités, Vol. XXXVI, Springer, Berlin, 2002, pp. 477-491.

[B-L] V.P Belavkin and J.M. Lindsay: The kernel of a Fock space operator II, Quantum probability and related topics VIII, World scientific, Singapore, 1993, pp. 87-94.

[Lin] J.M. LiNDSAY: The kernel of a Fock space operator I, Quantum probability and related topics VIII, World scientific, Singapore, 1993, pp. 271-280.

[Maa] H. MAassen: Quantum Markov processes on Fock space described by integral kernels, Quantum probability and related topics II, Lecture Notes in Mathematics Vol. 1136, Springer, Berlin, 1985, pp. 361-374.

[Mey] P.-A. MeYER: Quantum probability for probabilists, Lecture Notes in Mathematics 1538, Springer, Berlin, 1993.

[Pau] Y. Pautrat: From Pauli matrices to quantum Ito formula, Prépublication de l'Institut Fourier, 2002, available on www.math.mcgill.ca/ pautrat. 